\title{
Von der Dynamik des Selbermachens
}

\author{
Durch neue Technologien der Vernetzung und \\ Produktion zeichnet sich ein Regime nutzer- \\ getriebener Innovation ab. Es bietet einen \\ neuen Ansatzpunkt zur Genese und Diffusion \\ nachhaltiger Produkte und Dienstleistungen. \\ Ob diese Potenziale ausgeschöpft werden \\ können, ist jedoch eine Frage der Gestaltung. \\ Von Sascha Dickel
}

D as Leitbild der Green Economy basiert auf dem Paradigma der ökologischen Modernisierung, welches die Umweltdebatte nun bereits seit 30 Jahren prägt. Im Kontrast zu einer ökologisch motivierten Modernisierungskritik, welche einseitig auf die Schattenseiten der Industrialisierung für Mensch und Natur verweist, betont diese Theorie, dass die industrielle Moderne nicht nur für sie typische ökologische Probleme generiert, sondern zugleich auch die Mittel hervorbringen kann, ihre selbst erzeugten Probleme erfolgreich zu bearbeiten (Jänicke 2000). Ein zentrales Mittel sind aus der Perspektive der ökologischen Modernisierungstheorie Innovationen. Diese nehmen typischerweise die Form von neuen Technologien, Produkten und Dienstleistungen an.

\section{Nachhaltige Innovationen verdrängen Alternativen}

Für den Erfolg oder Misserfolg ökologischer Modernisierung ist zentral, ob es gelingt, bestehende ökologische Problemlagen durch Innovationen tatsächlich lösen zu können. Wie alle Innovation sind auch Innovationen, von denen man sich nachhaltige Effekte erhofft, mit dem Problem der sozio-ökonomischen Diffusion konfrontiert. Sie müssen nicht nur generiert werden, sondern auch gesellschaftlich akzeptiert und nachgefragt werden. Damit nachhaltige Innovationen tatsächlich transformativ wirken, sollten sie idealiter disruptiv wirken. Sie sollten also nicht nachhaltige Alternativen vom Markt verdrängen (Nill 2009).

Die Verantwortung für die Generierung und Verbreitung nachhaltiger Innovationen wird typischerweise Unternehmen (die neue Produkte und Dienstleistungen kreieren) und politischen Akteuren (welche die Rahmenbedingungen für Innovationen gestalten oder selbst Innovationen initiieren) zugeschrieben. Lenkt man den Blick jedoch auf Akzeptanz und Diffusion als notwendige Bedingungen von Innovationen, wird deutlich, dass Konsument/innen und Nutzer/innen eine Schlüsselstellung im Innovationsprozess zukommt. Diese Schlüsselstellung wird vonseiten der Innovationstheorie zunehmend erkannt und reflektiert.

\section{Die Öffnung von Innovationsprozessen}

„Open Innovation“ beschreibt einen neueren Innovationsansatz, der bestrebt ist, die Einsichten zur zentralen Rolle von Nutzer/innen in praktisch wirksames Handeln zu überführen. Im Kern geht es bei Open Innovation stets darum, den Innovationsprozess für externe Akteure zu öffnen, um deren Expertise möglichst früh in die Gestaltung von Innovationen einzubeziehen (Piller/Ihl 2009). Die Adressaten von neuen Technologien, Produkten und Dienstleistungen werden im Kontext von Open Innovation nicht länger als passive Konsument/innen betrachtet, sondern als „Prosument/innen“ (ein Kunstbegriff aus Produzent/innen und Konsument/innen), die eine mehr oder weniger aktive Rolle im Innovationsprozess einnehmen (Hellmann 2010).

Die besondere Bedeutung von Prosument/innnen für Innovationen erklärt sich aus ihrem spezifischen Wissen. Personen, die Adressat/innen und Abnehmer/innen von Innovationen sind, verfügen erstens über Selektionswissen. Das heißt über Wissen bezüglich der Motive, die bestimmten Kauf- und Nutzungsentscheidungen zugrunde liegen. Sie verfügen zweitens aufgrund von Nutzungserfahrungen über pragmatisches Wissen über den oftmals eigensinnigen Umgang mit Produkten und Angeboten. Die Erfahrungen hinsichtlich der sozialen Handlungsfelder und Alltagsroutinen, in die Innovationen erfolgreich eingebettet werden müssen, machen sie drittens zu wertvollen Inhabern von Kontextwissen.

Will eine Region zum Beispiel den öffentlichen Personennahverkehr (ÖPNV) neu gestalten, um ein alternatives Gegenangebot zum Autoverkehr zu schaffen beziehungsweise zu verbessern, ist es hilfreich zu wissen, was Personen zur Nutzung verschiedener Mobilitätsformen bewegt und welche Werte und Vorlieben dabei eine Rolle spielen (Selektionswissen). Ebenso wichtig ist es, zu erfahren, wie die konkreten Nutzungspraktiken von ÖPNV-Angeboten eigentlich aussehen (pragmatisches Wissen) und welche spezifischen Anforderungen etwa bei Berufspendlern und Besuchern bestehen, welche Einflüsse also angrenzende Handlungskontexte auf die Nutzung haben können (Kontextwissen) (Kropp/Beck 2011: 334-341). 


\section{„Bei der Gestaltung der Green Economy muss der Blick vermehrt auf die dezentrale Bottom-up-Ökonomie gerichtet werden."}

\section{Das Wissen von Nutzern}

Das Wissen von Nutzer/innen liegt typischerweise implizit vor, es handelt sich um „sticky information“ (Hippel 1994), die kaum durch oberflächliche Befragungen, etwa in Form quantitativer Marktforschung, extrahiert werden kann. Vielversprechender sind daher interaktivere Methoden der Nutzerintegration, in denen Prosument/innen selbst zu Innovationspartnern werden. Die Tiefe der Nutzerintegration ist bei solchen Arrangements dennoch begrenzt. Faktisch sind Prosument/ innen häufig weniger gleichberechtigte Mitgestalter/innen als vielmehr bloße Ideenlieferant/innen. In der Praxis zeigt sich, dass selbst gute Ideen von Nutzer/innen oft nicht umgesetzt werden, da sie den bestehenden Routinen von Organisationen zuwiderlaufen und/oder das Wissensmonopol der organisationsinternen Expert/innen bedrohen. Insbesondere Ideen zu nachhaltigen Lösungsansätzen, denen ein disruptives Potenzial innewohnt, da sie bestehende Geschäftsmodelle gefährden und bestimmte Produkte und Dienstleistungen überflüssig machen würden, sehen sich mit starken Beharrungskräften konfrontiert. Auf dem Weg in eine Green Economy ist somit offenbar nicht der Mangel an innovativen Ideen das entscheidende Problem, sondern vielmehr die bestehende Innovationskultur. Diese Kultur verfügt zum einen über unzureichende Mittel und Wege, auf diese Ideen zuzugreifen, und sie tut sich zum anderen schwer damit, externe Impulse im Rahmen der bestehenden institutionellen Strukturen tatsächlich umzusetzen (Kropp/Beck 2011: 341-346).

\section{Nutzergetriebene Innovationen}

Damit stellt sich die Frage nach alternativen Wegen der Generierung und Diffusion von Nachhaltigkeitsinnovationen, die zur Realisierung einer Green Economy dringend benötigt werden. Hierbei lohnt der Blick auf ein alternatives Innovationsregime, das sich gegenwärtig herausbildet und in dem die Rolle von Prosument/innen als gestaltende (und nicht nur ideenliefernde) Akteure in weitaus stärkerem Maße in den Vordergrund rückt, als dies typischerweise bei Arrangements der Nutzerintegration der Fall ist. In diesem emergierenden Innovationsregime stehen nicht die Innovationsaktivitäten etablierter politischer und ökonomischer Akteure im Vordergrund, sondern die
Ideen der Nutzer/innen selbst. Die Ermöglichungsbedingung dafür stellen neue Technologien der Vernetzung und Produktion dar. Diese technischen Innovationen werden zu Wegbereitern für soziale Innovationen, die ohne die transaktionskostensenkenden Effekte des (zunehmend mobilen) Internets kaum realisierbar wären.

Das vom Institut für ökologische Wirtschaftsforschung bearbeitete Projekt „Potenziale von neuen Technologien, dezentraler und personalisierter Produktion vor dem Hintergrund des Leitbildes kohlenstoffarmer Wirtschaft“ ist diesen neuen Dynamiken auf den Grund gegangen. Dabei zeigte sich eine enorme Vielfalt an Akteuren, Praktiken und Infrastrukturen nutzergetriebener Innovation. In den letzten Jahren haben sich verschiedene Formen dezentraler und kollaborativer Innovationsgenerierung und -gestaltung herausgebildet, deren Besonderheiten darin bestehen, dass sie an den bestehenden institutionellen Strukturen vorbeiführen. Crowdfunding-Plattformen wie Kickstarter oder Indiegogo bieten die Möglichkeit, Projekte und Produkte onlinebasiert zu finanzieren. Aus vielen kleinen Geldbeiträgen, die Nutzer/innen in Projekte investieren, die sie überzeugen, können so beeindruckende Summen entstehen, welche die Realisierung von Ideen ermöglichen, die ansonsten womöglich resonanzlos versiegen würden. In offenen Innovationslaboren, sogenannten FabLabs und Makerspaces, können Nutzer/innen ihre Ideen mit teils avancierten digitalen Fertigungstechnologien wie etwa 3D-Druck in die Realität umsetzen. Der Zugang zu den neuen High-Tech-Werkstätten ist grundsätzlich allen Interessierten möglich. Neue Onlineplattformen wie Etsy, Dawanda oder Shapeways ermöglichen den Vertrieb und Verkauf selbst gefertigter Produkte und bieten einen niedrigschwelligen Zugang zu Konsummärkten. In enger Verbindung zu diesen Phänomenen entsteht eine neue Kultur des Tauschens und Teilens, eine internetbasierte Sharing Economy, in der Nutzer über Apps wie „Whyown.It“ prinzipiell jedes Produkt leihen und verleihen können (Bauwens et al. 2012).

Wie gesellschaftlich anschlussfähig die Tendenz zum Teilen und "Selbermachen“ ist, zeigt sich an den stetig wachsenden Besucherzahlen der Maker Faire in Kalifornien, einer Mischung aus Messe, Festival und Konferenz, auf der sich die Szene der Bottom-up-Innovatoren Jahr für Jahr versammelt. Das Event zieht mittlerweile mehr als 100.000 Menschen aus aller Welt an.

Das implizite Wissen von Nutzer/innen kann durch die neuen dezentralen Vernetzungs- und Produktionsmuster prinzipiell wesentlich schneller und unmittelbarer in neue Technologien, Produkte und Dienstleistungen umgesetzt werden. Der „Umweg“ über etablierte Organisationen entfällt. Während im Paradigma der Open Innovation Prosument/innen als externe Partner von Unternehmen fungierten, treten Unternehmen im diesem alternativen Innovationsregime in den Hintergrund. Zum Teil beginnen sie ihre Rolle als Unterstützer, Plattformanbieter und Partner nutzergetriebener Integration neu zu definieren. 


\section{Die Dynamik des Selbermachens}

Insgesamt scheint es somit zunehmend relevant, bei der zukunftsfähigen Gestaltung einer Green Economy den Blick nicht nur auf die klassischen „big player“ zu richten, sondern auch und gerade der entstehenden dezentralen Bottom-up-Ökonomie vermehrt Beachtung zu schenken. Innovationen, die mit bisherigen nicht nachhaltigen Mustern brechen, sind hier womöglich leichter umsetzbar als in den Strukturen etablierter Organisationen und ihrer Netzwerke. Zudem können gerade Technologien dezentraler Produktion, wie 3D-Drucker, als Wegbereiter einer nachhaltigeren Wirtschaftsweise betrachtet werden, da sie lange Lieferketten überflüssig machen und eine Produktion vor Ort ermöglichen.

Gleichwohl kann nicht davon ausgegangen werden, dass nutzergenerierte Innovationen per se nachhaltiger sind als Innovationen, die in den etablierten Strukturen generiert werden. Vielmehr handelt es sich hier um ein neues Ensemble technischer und sozialer Innovationen, das sich immer noch auf der Stufe einer experimentellen Nischenpraxis befindet. Deren Zukunft ist ungewiss. Die Entwicklung des Internets von einem offenen und dezentralen System zu einem System, in dem eine überschaubare Anzahl mächtiger Akteure (wie Google und Facebook) den Ton angeben, verweist darauf, dass dezentrale Bottom-up-Strukturen durchaus wieder in zentral gesteuerte Topdown-Strukturen transformiert werden können (Morozov 2011).

Es wird daher zukünftig darauf ankommen, die Entwicklung des neuen Innovationsregimes weiter wissenschaftlich zu beobachten und aktiv mitzugestalten, um seine Nachhaltigkeitspotenziale auszuloten, auszuschöpfen und zu bewahren. Dabei stellt sich nicht zuletzt die Frage, welche Rahmenbedingungen und Infrastrukturen das neue Innovationsregime benötigt, um aus der Nische herauszutreten und einen Beitrag zu einer Green Economy leisten zu können.

\section{Literatur}

Bauwens, M. / lacomella, F. / Mendoza, N. / Burke, J. / Pinchen, C. / Léonard, A. / Mootoosamy, E. (2012): Synthetic Overview of the Collaborative Economy. Orange Labs und P2P Foundation. http://p2p.coop/files/reports/ collaborative-economy-2012.pdf.

Hellmann, K.-U. (2010): Prosumer Revisited. Zur Aktualität einer Debatte. Eine Einführung. In: Blättel-Mink, B. / Hellmann, K.-U. (Hrsg.): Prosumer Revisited. Zur Aktualität einer Debatte. Wiesbaden, VS Verlag für Sozialwissenschaften, S. 13-48.

Hippel, E. (1994): „Sticky Information“ and the Locus of Problem Solving. Implications for Innovation. In: Management Science 40, 4/1994, S. 429-439. http://web.mit.edu/evhippel/www/papers/stickyinfo.pdf.

Jänicke, M. (2000): Ökologische Modernisierung als Innovation und Diffusion in Politik und Technik: Möglichkeiten und Grenzen eines Konzepts. FFU-Report 01/2000. Forschungsstelle für Umweltpolitik, FU Berlin. http://www.polsoz.fu-berlin.de/polwiss/forschung/systeme/ ffu/publikationen/2000/jaenicke_martin_20004/rep_00-01.PDF.

Kropp, C. / Beck, G. (2011): Wie offen sind offene Innovationsprozesse? Von Nutzerrollen und Umsetzungsbarrieren. In: Defila, R. (Hrsg.): Wesen und Wege nachhaltigen Konsums. Ergebnisse aus dem Themenschwerpunkt „Vom Wissen zum Handeln - neue Wege zum nachhaltigen Konsum“. München, Oekom, S. 333-347.

Morozov, E. (2011): Back to the Roots. Cyberspace als öffentlicher Raum. In: Blätter für deutsche und internationale Politik 9/2011, S. 113-120.

Nill, J. (2009): Ökologische Innovationspolitik: Eine evolutorisch-ökonomische Perspektive. Marburg, Metropolis.

Piller, F. / Ihl, J.C. (2009): Open Innovation with Customers. Foundations, Competences and International Trends. Technology and Innovation Management Group: RWTH Aachen.

\section{AUTOR + KONTAKT}

Dr. Sascha Dickel ist wissenschaftlicher Mitarbeiter m Forschungsfeld Umweltökonomie und Umweltpolitik am Institut für ökologische Wirtschaftsforschung. IÖW, Potsdamer Str. 105, 10785 Berlin. Tel.: +49-30-884594-0, E-Mail: sascha.dickel@ioew.de, Website: www.ioew.de

\section{Briefe zur Interdisziplinarität}

Geistes-, Natur-, Ingenieur- und Sozialwissenschaften, Kunst \& Handwerk im Dialog

im oekom verlag, München. Mehr I nformationen unter

www. oekom. de/briefe-zur-interdisziplinaritaet
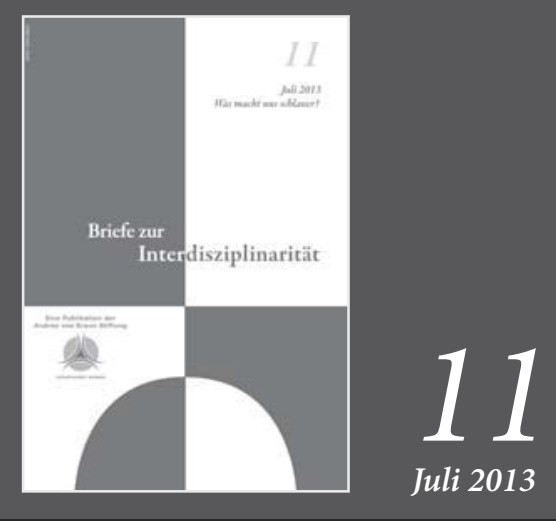
Copyright (C) 2013, IÖW und oekom Verlag. Die Nutzung des Artikels ist Abonnenten von Ökologisches Wirtschaften vorbehalten. Nachdruck und Vervielfältigung des Artikels einschließlich Speicherung und Nutzung auf optischen und elektronischen Datenträgern nur mit Zustimmung der Redaktion von Ökologisches Wirtschaften (http://www.oekologischeswirtschaften.de). 